\title{
New Initiatives are to be Enforced to Execute More Research on Thorough Degradation of Global Polymers Using Diversified Microbes/ Enzymes in the Eco-Friendly Way
}

\author{
Anju Kaushal* \\ Assistant Professor (Former), Environmental Sciences, SIET, Shiva Group of \\ Institutions, Bilaspur, H.P., India \\ *Corresponding Author: Anju Kaushal, Assistant Professor (Former), \\ Environmental Sciences, SIET, Shiva Group of Institutions, Bilaspur, H.P., India.
}

Received: February 17, 2020

Published: March 10, 2020

(C) All rights are reserved by Anju Kaushal.

\section{Abstract}

Plastic pollution is the crucial topic of concern at present and mainly $80 \%$ of polymers encompass the pollution. Because of inappropriate recycling, it is creating a detrimental impact on the ecosystems of our land and oceans. Many studies have been pursued on plastic biodegradation and found that the crystalline form of plastics actually degrades at different levels and it is a quite slow process. The microplastics are broken-down with exoenzymatic activities into smaller monomers and then easily get entered the cell wall of bacteria. These monomers then get assimilated inside to cells to form $\mathrm{CO}_{2}$ and biomass.

Plastic litter's accumulation happens largely in marine environment and there is no area in the sea which is not affected by human influence. The micro and meso plastics get eaten-up by marine animals, resulting the release of toxic chemicals. The bacteria Citrobacter and Kosakonia are found in the gut of worm Tenebrio molitor and identified to degrade polyurethanes and polystyrene. Polyurethanes, polyethylene, polyethylene terephthalate, polystyrene, polyvinyl chloride and polypropylene are the preeminent polymers to be used in our economy. A few fungi and bacteria have been identified for the partial degradation of polymers e.g. Themofifida, Thermomonospora, Ideonella sakaiensis, Pseudomonas putida, microspora, Cladosporium and other species. The enzymes involved in the hydrolysis are tannase, MHETase, cutinases and other hydrolases enzymes. The new biodegradable plastics have also introduced in the market as they are easily attacked by microorganisms to degrade them in eco-friendly way. The cold environment microorganisms degrade the plastics are, Pseudomonas sp, Shewanells, Psychrobacter Aspergillus, vibrio sp, Rhodococcus etc. The actual biodegradation process for cold environment is not systematically proven yet. Some pathogenic species of bacteria can spread through "Plastosphere", but their pathogenic evidence is still needed to verify.

The metagenomic studies on Chryseobacterium and Parapedobacter identify the high average novelty scores indicating the new species to be involved in polymer biodegradation process. The deconvoluted genome sequences report that biodegradation occurs in the oxidative pathways by oxidising the NAD+ and ADH by monooxygenase to produce methyl acetate and also by transformation of acetyl- CoA via acetone. BP8 community is first time discovered as the promising source for environmental biotechnology, which has the great phenotypic potential and catalytic capabilities to degrade polymers including xenobiotics. Putative metabolic pathway is discovered by a novel microbial landscape to breakdown PE-PU-A copolymers and xenobiotics. New strategies could be designed to reduce plastic pollution for better environmental quality. The current biodegradability standards are insufficient to identify the pragmatic approach and to compare it with in the same time frame in actual marine environment. There are so many ways, we still need to explore to get the realistic solution in identical conditions applied for Lab and outer natural environment.

Keywords: Biodegradation; Polymers; Polyethene; Enzymes; Microbes; Environment; Marine; Standards 
New Initiatives are to be Enforced to Execute More Research on Thorough Degradation of Global Polymers Using Diversified Microbes/ Enzymes in the Eco-Friendly Way

\section{Abbreviations}

PET: Polyethylene Terephthalate; PUR Or PU: Polyurethane; PE: Polyethylene; PS: Polystyrene; PVC: Polyvinyl Chloride; EC: Enzyme Class; MHET: Mono-2-Hydroxy Ethyl Terephthalate; NAD: Nicotinamide Adenine Dinucleotide; ADH: Alcohol Dehydrogenases; BP8: Microbial Consortium With Higher Novelty And Lower ANI Values; FAD: Flavin Adenine Dinucleotide; NADH: Reduced Form Of Nicotinamide Dinucleotide; 2-BE: 2-Butoxy Ethanol; DPGM: Dipropylene Glycol Methyl Ether; DPGB: Dipropylene Glycol Butyl Ether; PPG: 1-PEG-9 Lauryl Glycol; PEG: Polyethylene Glycol; HMM: Hidden Markov Model; ANI: Average Nucleotide Identity; NMP: Novel Metabolic Pathway; $\mathrm{CO}_{2}$ : Carbon Dioxide; $\mathrm{CH}_{4}$ : Methane; $\mathrm{N}_{2}$ : Nitrogen; $\mathrm{H}_{2} \mathrm{O}$ : Water; ISO: International Organization For Standardization; ASTM: American Society For Testing And Materials; EN: European Standards; ATP: Adenosine Triphosphate

\section{Introduction}

Plastics have been in use, in our day to day life, for last 100 years, and its large-scale production started in 1950 [19]. 350 to 400 million tons of plastics are being produced each year and widely used in the global economy. Due to poor recycling, millions of tons get accumulated annually in terrestrial and marine environments. Consequently, it causes the adverse effect on the all ecosystems. Microplastics, in particular, are our big health concern.

The current microbial research has shown the extent of degradation of plastics by microorganisms and enzymes available on the high- molecular-weight polymers of polyethylene terephthalate (PET) and ester- based polyurethane (PUR). These high molecular weight polymers are used to make plastics, still no enzymes available to breakdown them fully. Together these polymers are comprised of the $80 \%$ of annual plastic production.

This review explains the research on various microbes and enzymes acting on these polymers. Metagenomic studies are done to design the more potent microbial communities to degrade plastics. Biologically dark matter studies, could be the future programs where the unidentified DNA and protein/ genome and proteome may refer to the un-isolated microorganisms, could have capability to degrade the polymers. These approaches could discover the novel and competent microbes and enzymes, having the required qualities to deliver the environmentally friendly solutions to degrade the human made polymers. These studies can open the urgent microbial science initiatives help reaching to the desired solutions.
Photodegradation and weathering are the main forces for initial breaking of plastic, in to the micro and nano forms, enters the food chain and end up in our intestines. However, the fate of these microplastics has yet to be determined. This removal of plastic by using enzymes and microbes is main focus area of research recently. Plastic is stable in oceans and soil. It has been quite short time for bacteria to evolve and produce relevant enzymes, since the production of plastics have been introduced first time in 1950. Plastics can frequently mixed with solubilizers and other chemical agents to alter their physical and chemical properties. These compounds target the microbial degradation and may interferes with the degradative enzyme activities. It is assumed that the initial larger polymers initially degraded by the exoenzymes into smaller subunits (monomers, dimers, and multimers) and these get taken up by microbial cells. Inside the cells they get degraded by biological pathways to yield energy and/ or serve as building blocks for catabolism or metabolism.

The high molecular weight of fibers, the strong C-C bonds, and the extremely hydrophobic surface, is very difficult to be attacked by enzymes. This made the degradation process very slow. The amorphous and crystalline forms of high molecular weight polymers, generally degrades at different levels.

Although plastic materials constitute an integral part of the global economy, the issues associated with their extensive application cannot be ignored. Accumulation of plastic litter occurs in the marine environment mostly, where it is hard to find any area that is unaffected by human influence.

\section{Plastic and its interactions with environment}

Plastic debris are found on the surface and also in deep Oceans. It is mainly found in forms of microplastics $(<5 \mathrm{~mm})$ and microplastics $(<5 \mathrm{~cm})$. A crucial obstacle is the high molecular weight can't be transported across the cellular membrane of microorganisms. Thus, the long-chain polymers have to be depolymerized to smaller monomers before they cross the cell membrane, followed by the assimilation by the intracellular metabolism.

Microplastics can spread fast and rapidly and reach to the places which are far away from the common pollution sources, hence affecting the untouched places even (a study in Antarctic marine). These contaminations have the significant impact on marine fauna due to entanglement, suffocation, and disruption of digestion in 
New Initiatives are to be Enforced to Execute More Research on Thorough Degradation of Global Polymers Using Diversified Microbes/ Enzymes in the Eco-Friendly Way

birds, fish, mammals and turtles [11]. Places located far away from pollution sources are also affected by plastic wastes. There is also a concern that it is a source of toxic chemicals such as polychlorinated biphenyls or phthalates and bisphenol A [8]. According to research of the Alfred Wegener Institute, Helmholtz Centre for Polar and Marine Research, around 1506 species are affected by the litter [45].

To understand pollution in marine environment that oceans not only accumulate plastics at certain points but also transfer them to distant virgin regions such as the Arctic and Antarctic. Moreover, the interaction between marine microorganisms and plastic waste progresses to the oceans. The information has also been collected on plastic degrading bacteria in cold marine habitat. Plastic hoards in the sea at some points and the biomass is accumulated on the floating plastic called biofouling. It involves in five main steps: adsorption, immobilization, consolidation, microfouling and macrofouling. In the deep-sea waste stays for longer, but superficially exposed to sun and degradation process starts. The large plastic particles affect the fish and birds, depending upon the concentration, pieces of microplastic can represent a big threat to the organisms of lower trophic levels such as zooplankton and muscles. They ingested it and egested with their fecal pellets. As a result, these pellets are the source of food for other marine organisms, constituting a vector for faster vertical transport.

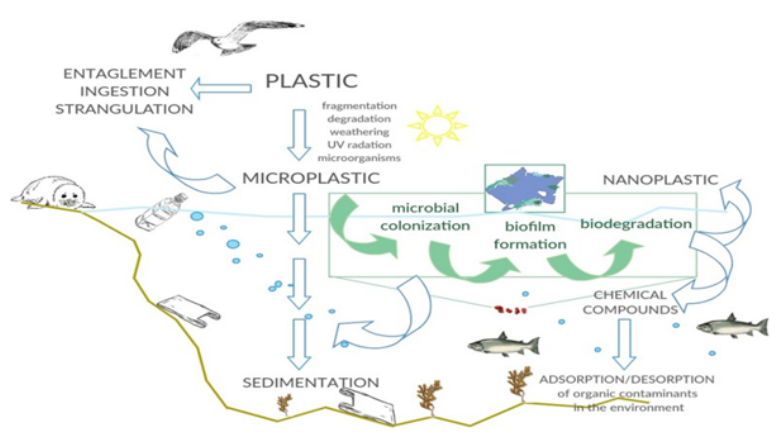

Figure 1: Interactions between marine microorganisms and microplastics in marine environment.

Source: https://www.ncbi.nlm.nih.gov/pmc/articles/ PMC6132502/

Invertebrate microbiome as a possible source of plastic degrading bacteria

Convincing evidence of mealworm, Tenebrio molitor $L$. to digest the styrofoam provided by Yang and colleagues. The digested $50 \%$ of styrofoam to convert it into $\mathrm{CO}_{2}$ and the residual Styrofoam was found in the faeces. Carbon labelling studies showed that polysty- rol implied that carbon compound was preferentially used to build lipids. A wax moth, Galleria mellonella, was shown to degrade PE by putting holes in it. Bacillus sp from Indian mealworm could digest the polyethylene using their gut bacterium $[47,48,49]$. A related study by identifying bacteria associated to genera Citrobacter and Kosakonia degraded the PE and PS in the guts of Tenebrio molitor [7].

\section{Microbial and enzymatic degradation of Polymers}

Polyurethane (PU), Polyethylene (PE), Polyethylene terephthalate (PET), polystyrene (PS), polyvinylchloride (PVC), and polypropylene. are the polymers mainly used in our economy.

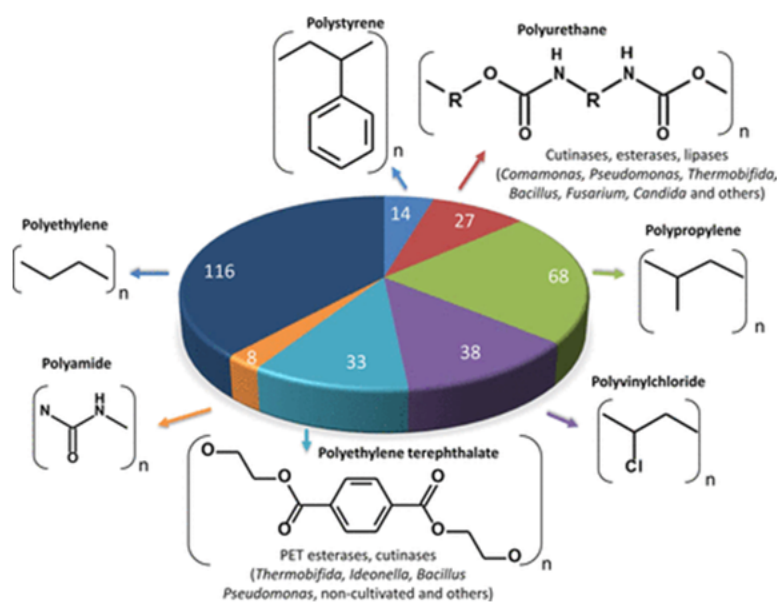

Figure 2: Polymers important to our economy.

Source: https://aem.asm.org/content/85/19/e01095-19.

\section{Polyethylene terephthalate (PET)}

Polyethylene terephthalate (PET) is mainly used for production of bottles, foils and fibres. PET is polar and linear polymer of repeating unit of terephthalic acid and ethylene glycol. PET is thermoplastic and partly crystalline. The annual production of PET is exceeded 30 million tons in 2017.

A few bacteria and fungi have been discovered for the partial degradation of PET to oligomers and monomers. All the hydrolases have very low turnover rate. Most of the bacterial isolates belong to the gram-positive phylum Actinobacteria, genera Thermobifida and Thermomonospora. The enzymes involved in the degradation are PET hydrolase and tannase, MHETase are specific serine hydrolases, e.g. cutinases (EC3.1.1.74), lipases (EC3.1.1.3). and carboxylesterases (EC 3.1.1.1). The $\alpha / \beta$ - hydrolase fold and catalytic triad is composed of serine, histidine and aspartate and also contain several disulphite- bonds of cysteine residue promotes thermal stability and specific binding to PET, e.g. PETase from Ideonella sakaiensis. 
New Initiatives are to be Enforced to Execute More Research on Thorough Degradation of Global Polymers Using Diversified Microbes/ Enzymes in the Eco-Friendly Way

Another important enzyme MHETase is secreted by I. sakaiensis genome codes, which is similar to tannases capable of degrading mono ( 2-hydroxyethyl) terephthalic acid(MHET). MHET is hydrolysed by MHETase enzyme and used for bacterial metabolism. I. sakaiensis belongs to phylum Betaproteobacteria and order Burkholderiales.

3D structure of PET ase is similar to cutinases. The majority of functionality verified PET hydrolases containing C-terminal disulphite- bond, promoting thermal and kinetic stability $[9,17]$. In total 4 MHET moieties are bound to the protein subsites, ester bond to be cleaved between subsites next to the catalytic serine. MHET is further hydrolysed by ethylene glycol and terephthalic acid. This leads the enzymes exclusively confers substrate specify and activity towards MHET, with $\mathrm{k}_{\text {cat }}$ of $11.1 \pm 1.4 \mathrm{~s}^{-1}$ [39].

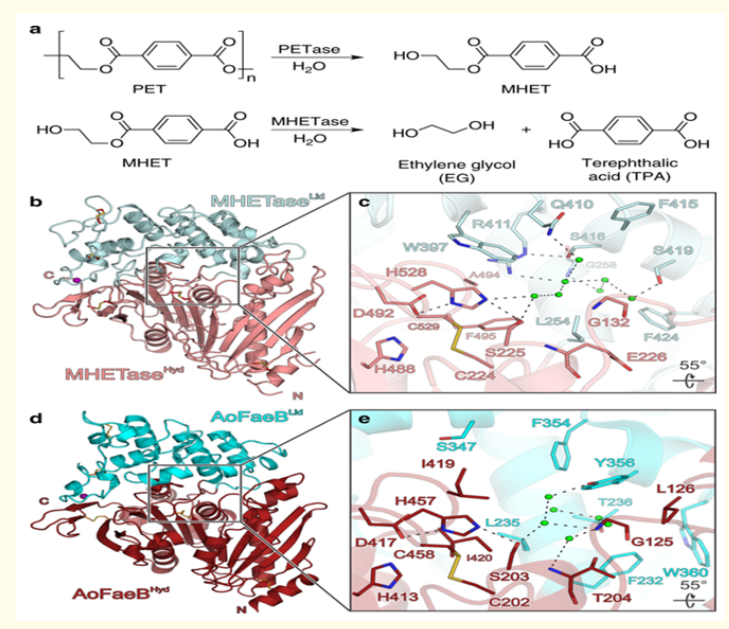

Figure 3: The structure of I. sakaiensis MHETase displays a bipartite domain architecture. a) I. sakaiensis PETase and MHETase degrade PET to terephthalic acid and ethylene glycol. Side products are not shown. b) MHETase structure with the $\alpha / \beta$-hydrolase domain (MHETaseHyd) colored in salmon and the lid domain (MHETaselid) in light blue. Disulfide bonds are represented by sticks. c) Close-up view of the MHETase catalytic triad d) A. oryzae FaeB (PDB-ID: 3WMT), $\alpha / \beta$-hydrolase domain (AoFaeBHyd) in crimson red, lid domain (AoFaeBLid) in cyan. e) Close-up view of the AoFaeB catalytic triad, oxyanion hole and the water molecules in the substrate-binding site. Dashed lines indicate hydrogen bonds, rotation angles relate to the overview. Interacting residues are shown as sticks and colored by atom type. Carbon-as given for the respective molecule; nitrogen-blue; oxygen-red; sulfur-yellow. Water oxygens are shown as green spheres. Calcium is shown as purple sphere.

A novel carboxylic ester hydrolase identified as with polyester (PET) degrading activities in the marine bacterium Pseudomonas aestusnigri VGX014T [26]. PET ase represents the best explored and studied class of enzymes for the hydrolysis of synthetic polymers.

\section{Polyurethanes}

Polyurethanes PUR is produced as 27 tons annually taking a fifth rank in synthetic polymers [41]. Its carbamide units with aromatic ring structures has a further impact on its physical and chemical properties. It is widely used synthetic polymer for the production of foams, insulation materials, textile coating, paints etc.

Gram negative Betaproteobacteria from genus Pseudomonas is associated with PUR degradation activities. The enzymes PueB and PueA lipase from Pseudomonas Chlororaphis help degradation of PUR with hydrolases which is tightly regulated by seven genes affiliate with seven open reading frames [24,26].

Pseudomonas protegens strain Pf-5, and Pseudomonas putida use the same mechanism of carbon catabolite control with lipase genes pueE and pueB. P. putida degrades PUR at high rate. Comamonas acidovorans TB-35 strain produces an enzyme esterase PudA, 62 KDa monomer, having hydrophobic domain to bind PUR to degrade it, and releases diethylene glycol and adipic acid at $45^{\circ} \mathrm{C}$ at pH6.5 [1]. Bacillus subtilis and Alicycliphilus sp are also isolated to degrade PUR.

Different enzymes from Pseudomonas sp showed significant activity by clearing the zones in agar plates containing colloidal PUR. The other assays PUR based NMR and Infrared assay showed significant degradation [5].

Thermobifida-derived cutinases are the lipolytic enzymes have shown activity on 78 different substrates including terephthalate and PUR [32].

Fungus Pestalotiopsis microspora, with its metallo-hydrolases, is responsible to degrade PUR. The other fungi are identified to degrade PUR as Fusarium solani, Candida ethanolica, Candida rugosa, Cladosporium pseudocladosporioides, C.tenuissimum, C. asperulatum, C. montecillo, Aspergillus fumigatus, A. flavus and Penicillium esterases. These species produced the enzymes were active on the ester linked PUR, but not on polyurethane ethers.

\section{Polyethylene}

Polyethylene (PE) are the long chained high and low-density polymers. It is used in the packaging and more than 100 million tons of PE are produced every year [26,34].

PE degradation is affiliated by microorganisms e.g. Pseudomans, Ralstonia, Stenotrophomonas, Rhodococcus, Staphylococcus, Streptomyces, Bacillus, Aspergillus, Cladosporium, Penicillium etc. The 
New Initiatives are to be Enforced to Execute More Research on Thorough Degradation of Global Polymers Using Diversified Microbes/ Enzymes in the Eco-Friendly Way

invertebrates' gut microbes support PE degradation. Degradation is measured by Fourier transform of infrared spectroscopy (FTIR).

Not much studies are deposited about the PE degrading enzymes and biochemical reaction. Except an enzyme laccase identified in Penicillium involved in PE breakdown [42].

Polyamide

Polyamide (PA) are the polymer of amide bonds linked aliphatic, aromatic and semi aromatic units e.g. nylon and kevlar. Polyamides have applications in textile, automotive, carpets and sport wear [40]. To date no enzyme were discovered to fully degrade polymers. Flavobacterium sp strain K172 was the first bacteria to act on nylon and later renamed as Achromobacter guttatus K172 and again renamed as Arthrobacter sp strain K172. Its hydrolases and aminotransferases genes are located on an accessary plasmid, pOAD2 [28,33].

The three main enzymes are invoved as cyclic dimer hydrolases (NylC), dimer hydrolases (NylB) and the endo type oligomer hydrolase (NylC). NylC a typical esterase structures with $\beta$ - lactamase fold. Once Oligomers are hydrolysed followed by the action of different aminotransferases on monomers to be metabolized. Arthrobacter cariies genes as nylD1 and nylE1 responsible for secondary 6-aminohexanoate metabolism. Aminotransferase catalyses aminohexanoate into adipate semialdehyde. It uses $\alpha$ - ketoglutarate, pyruvate and glyoxylate as amino acceptors and generate glutamate, alanine and glycine. The second enzyme, the adipate semialdehyde dehydrogenase (NylE1) convert adipate semialdehyde into adipate $[43,44]$.

Diverse marine bacteria e.g. Bacillus cereus, B. sphaericus, Vibrio furnissi and Brevundimonas vesicularis were identified as nylon degraders. Mn-dependent peroxidase enzymes from white rot fungus can disintegrate the nylone-66 membranes. Enzyme from Pseudomona aeruginosa, 6-amino hexanoate linear dimer hydrolase utilize the 6-amino hexanoate-dimer as a carbon and nitrogen source [29].

\section{Polystyrene}

Polystyrene (PS) polymers consists of styrene monomers, used for packaging. 14 million tons were produced in the year 2016. Unfortunately, there is no enzyme known that can degrade high molecular weight polymer. White rot fungus Gloeophyllum striatum DSM9592 and Gloeophyllum trabeum DSM 1398 caused substantial depolymerization after 20 days. The strain caused 50\% reductions in molecular weights. But the enzymes involved in the degradation studies are still to be elucidated. Weight loss have been caused by the degradation of chemical additives [30].

Styrene degradation in bacteria is well studied in Pseudomonas, Rhodococcus, Corynebacterium and others. Under aerobic conditions the styrene is oxidised into two paths:

1. Attacking the vinyl side chain

2. An unspecific aromatic ring, forming primarily the intermediate 3-vinylcatechol, phenylacetic acid, and 2-phenylethanol. These intermediates are channelled into Krebs cycle after ring cleavage.

Vinyl side chain is degraded by three enzymes: a styrene monooxygenase, a styrene oxide isomerase, and a phenylacetaldehyde dehydrogenase. Styrene monooxygenase attacks the vinyl side chain to release epoxy styrene, which results isomerization to form phenylacetaldehydes. This is oxidized to phenyl acetic acid through the involvement of a dehydrogenase. In P. putida, the phenylacetic acid is activated to phenylacetyl-coenzyme A ( $\mathrm{CoA})$ and then subjected to $\beta$-oxidation to yield acetyl-CoA, which directly fed into Krebs cycle. The relevant gene cluster designated as $\operatorname{sty} A B C(D)$. The sty $A$ and $s t y B$ genes code for the styrene monooxygenase complex. Monooxygenase has 2 parts of flavoproteins to catalyse NADH and FAD - dependent epoxidation to styrene to styrene oxide. The styB functions as FAD reductase to transfer the electron from NADH to FAD and then to styA. styC gene codes for the styrene isomerase and styD for phenyl aldehyde dehydrogenase gene [9,35]. The expression of the conserved cluster is regulated by LysR-type regulators [36,37] (Figure 4).

The styrene ring is cleaved by the initiation of a dihydroxylation of the aromatic ring. The reaction is catalysed by a 2,3-dioxygenase and followed by a 2,3-dihydrodiol dehydrogenase to form two key products cis-glycol and 3-vinylcatechol. The latter is then transformed in acrylic acid, acetaldehyde, and pyruvate. The pathway is rather unspecific for the general degradation of various aromatic compounds [46]. 


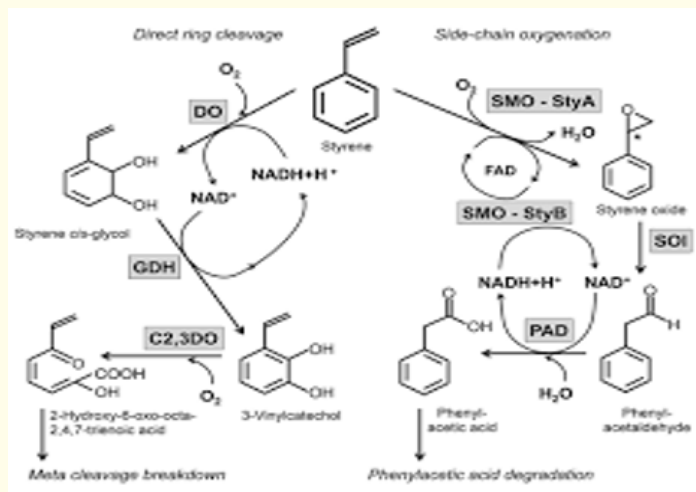

Figure 4: Styrene metabolizing cascade.

Credit: Frontiersin.org.

Phenylacetaldehydes are the interest of different industries e.g. fine chemicals and pharmaceutical compounds. This can be a starting material for fragrances, flavours, pharmaceuticals, insecticides, fungicides and herbicides. Recent studies showed that Pseudomonas putida, Rhodococcus zopfii, and other gram negative sp convert polystyrene into biodegradable polymer polyhydroxyalkanoate. This approach involved in a pyrolysis of polystyrene to styrene oil and then to polyhydroxyalkanoate. This process is not feasible in large scale, as the pyrolysis process runs at $520^{\circ} \mathrm{C}$ and energetically it is demanding $[36,37]$.

Polyvinylchloride and polypropylene

Polyvinylchloride (PVC) and polypropylene (pp) are both important polymers. PVC is the third most polymer produced frequently. PP and PE are produces at higher levels. There is hardly any specific information on the microbial degradation of these polymers except the weight loss experiments using mixed microbial species, despite the fact they are produced largely. So, no specific pathway is known for enzymatic actions for the degradation of these polymers.

Metagenomic and BP8 microbial community for biotechnology strategy

A hidden Markov model (HMM) is applied for some complementary approaches to discover accurate activities on motif based large scale genomic and metagenomic studies to recover a data sequence which are not immediately available. The bioinformatics studies for alignment of bio sequences and sequence classifications has been developed for the presence of a potential PET hydrolases. Using this approach more $>800$ potential PETases were identified in bacterial and archaeal genome and metagenomes. Various functionally active enzymes were discovered (e.g. PET2, PET4, PET6, and PET 12), and they are globally distributed in marine and terrestrial metagenomes [10].

The deconvoluted genomes of Paracoccus sp BP8 and O. intermedium BP8.5 showed low novelty scores and high average nucleotide identity ANI value compared to their closet phylogenetic relatives. In contrast, Chryseobacterium sp BP8.2, Parapedobacter sp BP 8.3, and the microbacteriaceae bacterium BP 8.4, showed high novelty scores and low ANI value $(<95 \%)$ indicating the new species.

BP8 microbial community exhibit variable cell-surface interactions that could be involved in biodegradation process. BP8 metagenomic analysis allowed to identify known additives and PU degrading enzymes and to propose new activities and metabolic pathways involved in biodegradation. The genes encoded the enzymes.

The BP8 Microbial Community exhibit Vvriable cell-surface interactions that could be involved in biodegradation process. BP8 metagenomic analysis allowed to identify known additive and PU degrading enzymes and to propose new activities and metabolic pathways involved in biodegradation. The genes encoding enzymes identified in deconvoluted genomes. Genes reported from A. denitrificans and Paracoccus sp is a BP8 genome. Paracoccus utilized $\mathrm{N}$ - methyl pyrrolidone a novel metabolic pathway NMP, as carbon source. IP biodegradation occurs by oxidative pathways in $P$. denitrificans GH3 and Gordonia sp TY-5. In these strains, IP is transformed by $\mathrm{NAD}^{+}$-dependent secondary $\mathrm{ADH}$ into acetone that is oxidized by a specific monooxygenase to produce methyl acetate, which is transformed to acetic acid and methanol [18] The possibility that IP degradation occurs by transformation to acetyl-CoA, via acetone in BP8 consortium is supported by the observation that in the Paracoccus sp. BP8 genome, a gene encoding an ADH (RQP05888.1), homologous to the Gordonia sp. TY-5 adh2, and genes encoding the acetone carboxylase subunits (RQP05866.1, RQP05867.1, RQP05889.1) are contiguously located. Adjacent to these genes, a sequence encoding a sigma-54-dependent transcriptional regulator (RQP05868.1) was observed, suggesting an operon-like organization. This presumptive IP degradative operon 
New Initiatives are to be Enforced to Execute More Research on Thorough Degradation of Global Polymers Using Diversified Microbes/ Enzymes in the Eco-Friendly Way

has not been described in any other bacteria. Degradation of 2-BE, DPGM and DPGB has not been reported in bacteria.

By using different analytical techniques, the BP8 community is demonstrated to attack the main functional groups of the PE-PU-A copolymer; from the more enzymatically susceptible ester bonds, present in acrylate and carbamate, to the more recalcitrant $\mathrm{C}-\mathrm{C}$ from aliphatics and aromatics, $\mathrm{C}-\mathrm{N}$ from urethane, and $\mathrm{C}-\mathrm{O}-\mathrm{C}$ from ether bonds of PPG. The biodegradative activity of BP8 community on the additives PE-PU-A is presented by using PolyLack* on its phenotypic potentials encoded in its metagenome. Hi-C proximity ligation-based technology allowed to define, with high confidence, what genes belong to each of the different species of BP8, especially in poorly represented species.

BP8 microbial community is a promising source for environmental biotechnology strategy. This has provided the deep understanding of the biodegradative activity of a landfill community capable of PU and xenobiotic degradation. Its outstanding phenotypic potential and catalytic capabilities displayed to cleave different recalcitrant groups, proposing the metabolic pathways involved in xenobiotic degradation.

Species specific enzymatic reaction could be exploited. Some more possibilities by assembling the specific consortia for increased PU degrading ability, the overexpression of additives or PU degrading proteins to be used in environmental biotechnology strategy for the waste treatment, and the development of bioenzymes for novel applications. This community is a promising source to contribute to mitigate anthropogenic plastics and xenobiotics pollution, for achieving better environmental quality.

The datasets generated for this study can be accessed from GenBank under Bio Project Accession number: PRJNA488119 [26].

By applying in silico genome mining approach, a cutinase from Pseudomonas pseudoalcaligenes (PpCutA) and a putative lipase from Pseudomonas pelagia (Ppela Lip) were discovered as potent enzymes to act on polyester in general. Recombinants of PpCutA and PpelaLip activity together on variety of polyesters, polyoxymethylene terephthalate [36]. Another species notably belong to biotechnologically novel genus Pseudomonas is named as Pseudomonas pertucinogena [11].

Metagenome derived esterases MGS0156 and GEN0105, used to hydrolyse prolactic acid, polycaprolactone and bis(bezoyloxyethyl)- terephthalate as well. MGS0156 with $\alpha / \beta$-hydrolase lid and a highly active hydrophobic site [20].

Itzel., et al. [26] characterized the biodegradation process of recalcitrant plastic PE-PU by microbial communities. They provide a comprehensive chemical and physical evidences of the capacity of a selected landfill microbial community to degrade the aromatic PE-PU-A copolymer and the introductory xenobiotic additives. The phenotypic and structural potential are analysed by Hi-C proximity ligation technology. They have identified a novel microbial landscape that can deal with PE-PU-A and xenobiotics additives degradation and proposed a putative metabolic pathways/ and gene regulator that can account for these capabilities. This is the first metagenomic analysis of polyurethane- enriched landfill community. Understanding these pathways help design the strategies that contribute to mitigate plastics and xenobiotics pollution to achieve the better environmental quality.

\section{Standards for biodegradation and their drawbacks}

Biodegradation is considered to occur after the physical and chemical degradation or abiotic degradation. It is summarized in four essential steps:

- The biofilms growing on plastic surface increase the pore size, and microorganisms release acids to modify the $\mathrm{pH}$ which weakens the plastic matrix.

- Extracellular enzymes like oxygenase, lipase, esterase, depolymerases etc. on the polymer surface, resulting in bio - fragmentation of polymers into oligomers and then monomers which can be assimilated by the cells.

- Oligomers $<600 \mathrm{kDa}$ to be integrated inside the cells to be used as carbon source, thus increasing the biomass.

- Mineralization is resulting in the excretion of completely oxidized metabolites $\left(\mathrm{CO}_{2}, \mathrm{~N}_{2}, \mathrm{CH}_{4}\right.$, and $\left.\mathrm{H}_{2} \mathrm{O}\right)$.

A degradation by number of microbes rather than a single species are necessary to degrade recalcitrant plastic [43]. The degradation timescale, characterization and fate of the same is fundamental, but it is still an unanswered question yet.

Under aerobic conditions, they impose $60-70 \%$ conversion from plastic to $\mathrm{CO}_{2}$ over a three months' time period (ASTM D669109), six months' time (ASTM D7473-12), or 24 months' time (ISO 18830, ISO 19679, ASTM D7991-15). Under anaerobic conditions the biodegradation is $5-7.35 \%$, characterized by specific standard 
New Initiatives are to be Enforced to Execute More Research on Thorough Degradation of Global Polymers Using Diversified Microbes/ Enzymes in the Eco-Friendly Way

ASTM D5511 in 15 days period, but these standards apply to the marine environment.

ASTM D 6400 - For compostable plastic product Labelling.

ASTM D6868- For plastic coatings and bindings that it is biodegradable.

EN 13432 - Harmonised European standard to find compostable plastic after 12 days and completely biodegradable after six months.

Biodegradation of plastic is characterized by time requires to achieve mineralization under specific / controlled conditions, to convert plastic into biomass $+\mathrm{CO}_{2}$. However, these tests can't provide ready results.

\section{Standardization issues}

It is found out that these standards are insufficient to predict degradability realistically in marine environment, and underestimate the time required as compared to the natural ecosystem [22].

The number of standards and test methods for measuring the biodegradability of polymers within aquatic environments have been developed, several issues can limit their reliability when attempting to in situ rates of biodegradation. These uncertainties originating from:

1. Inoculum preparations and test conditions.

2. A lack of specific guidelines for employing different test materials.

3. Insufficient statistical replication.

4. Lack of suitable procedure for unmanaged aquatic environments.

5. Deficiencies in toxicity testing and our understanding of the wider impact of plastic litter on aquatic ecosystem.

Knowledge gaps

1. Comparability of laboratory- based data to the pattern of biodegradability in an open environment.

2. Absence of biodegradability standards and test methods for unmanaged aquatic environments.

3. Lack of wider laboratory and field - based research into polymer biodegradation within several aquatic environments.
4. Toxicity tests and research into the wider impact of plastic materials on aquatic ecosystems.

\section{Alternative methods to detect biodegradability}

Measurements of biodegradability is commonly based on the biologically oxygen demand/ or the evolution of $\mathrm{CO}_{2}$ and / or $\mathrm{CH}_{4}$. An alternative method based on ATP measurements has been proposed to assay the biodegradability of 'oxo-degradable' polymer films [3]. The indicators to determine the biodegradability is determined by changes in the physical properties.

- Evaluation of plastic material to fungi and bacteria, by changes in the visual appearance- ASTM G 21-15, G22-76 and D7473-12 (ASTM 2015, ASTM 1996, ASTM 2012).

- $\quad$ Changes in the mass are monitored in the withdrawn testthe ASTM D5247-92 and D6003-96, and ASTM D7473-12 (ASTM 2012, ASTM 1992, ASTM 1996)

- $\quad$ Changes in the mechanical properties are additionally assessed as apart of ASTM D5247-92(ASTM 1992).

The biodegradation step is divided into three phases. In the early phase the gases $\mathrm{CO}_{2}$ and $\mathrm{CH}_{4}$, and their evolution is negligible stated as lag phase, subsequently exhibited a substantial increase (biodegradation phase), and followed by plateau phase when biodegradation is completed [31]. Materials exhibiting loss of $90 \%$ of mass are often assumed to be nearly completely biodegradable.

Future glimpses

Since the plastics began to be manufactured in 1950s, so the naturally evolved microbes and related enzymes are limited. We need to increase the diversity of microorganisms which are capable to biodegrade polymers completely:

1. The main barrier is the identification of the organisms effectively acting on the most dominant polymers, addressing the high molecular weight, highly robust polymers and their crystalline structures. Diversity of microbes and enzymes acting on polymers is still very limited.

2. Implementation of series of enzymes in the process that would allow the polymer degradation in polluting environmental area, is a great challenge for the future microbiologists. The current cultivation technologies have not yet resulted to find out the effective enzymes. Implementation of enzymatic -process can be an ultimatum for microbiologists at this stage. 
New Initiatives are to be Enforced to Execute More Research on Thorough Degradation of Global Polymers Using Diversified Microbes/ Enzymes in the Eco-Friendly Way

3. Cutinase enzyme degrade the cutin and some PET like polyesters. More work needs to be done as mentioned in following points:

- Optimization of the enzyme level work better.

- High activity of mutated enzymes could erode crystalline PET, as enzymes evolve.

- Stability of enzymes at higher temperature to improve the binding affinity for PET and break the bonds

- $\quad$ Process optimization is needed to stop the enzyme aggregation to enhance the performance and strategically putting the carbohydrate groups to prevent aggregation.

4. The global metagenome and dark matter proteins studies offer the promising source to find out effective biocatalysts, can help to find diversity in the non-cultivated microorganisms. A structure of function- based assays for the detection of highmolecular-weight-polymer-active enzymes is also important in parallel.

5. The overall technology by microbial degradation needs to be standardized and optimized. Development and implementation of enzyme function- based assay is also important. Commercially available polymers and films used as substrate contains lots of impurities like additives, plasticizers, other biodegradable impurities like phthalates, which are much more easily broken down than the actual bone. Hence, it interferes with results and likely to lead to the identification of false positives.

6. Studies can also be performed on genetic engineering of plastosomes and cellulosome like structures in bacteria. In order to utilize the cells nutrients into energy to regulate the metabolisms. These set of reactions can be carried out in the Citric acid cycle/ or Krebs cycle. As a result, they can attack on the crystalline fibres would be an excellent project.

7. The more realistic short- term goals would be to work on highly active enzymes to be used in the textile industries, could significantly reduce the plastic pollution on yearly basis.

8. Creation of microorganisms using synthetic biology would be used to make value added compounds from the plastic waste is a future challenge. These will improve circular use of plastic. Monomers and dimers after degradation can be transformed in to value added biodegradable polymers.
9. By noticing the complex problems with polymers, the new research initiatives on "Polymer and Oceans" should bring forward, where the scientists from different field e.g. physics, chemistry, biology and, biotechnology backgrounds, could work in collaboration to find out the solutions to face this environmental problem and resolve it for the society.

10. There are still significant gaps regarding the plastic biodegradation in natural environment. There are some challenges regarding the risky outcomes through the formal standards should be considered in this context, and these are as:

- The standards which perform the safe environmental credentials for plastic films and bags that might be unintentionally littered.

- The laboratory test results should be designed to simulate the conditions in open environment.

- Working on the strengths and weakness of the current biodegradability standards and specifications for plastic limit.

- Development of biodegradability standards specifications and compliance for new generation plastic bags claiming to be biodegradable in all environments.

- $\quad$ Are all open environments equally well supported by biodegradability standards and scientific knowledge, or are some better served than others?

11. Breakdown tool for plastics recycling by microbes is too slow to implement this technology for immediate application. A bio-cycling process can help discover the productive way to harvest and reuse monomer products. In monomer isolation they should make the enzymes which can be stable at higher temperatures.

12. Optimization process is expensive for biotechnological processes requires more investments. It is tough to compete with low price of monomers at present. Changing attitude and changing demands from consumers could create the balance in favour of recycling methods that use biocatalysts rather than the chemical catalysts if it reaches to the competitive cost.

\section{Conclusions}

The conclusion of this review is that current international standards and regional test methods are insufficient in their ability to realistically predict the biodegradability of carrier bags within wastewater, inland waters (rivers, streams and lakes) and marine

Citation: Anju Kaushal. "New Initiatives are to be Enforced to Execute More Research on Thorough Degradation of Global Polymers Using Diversified Microbes/ Enzymes in the Eco-Friendly Way". Acta Scientific Microbiology 3.4 (2020): 97-108. 
New Initiatives are to be Enforced to Execute More Research on Thorough Degradation of Global Polymers Using Diversified Microbes/ Enzymes in the Eco-Friendly Way

environments. This is due to several shortcomings in existing test procedures, the absence of relevant standards for the majority of unmanaged aquatic habitats, as well as a paucity of wider research into the biodegradation of polymers within these environments.

Could nature get rid of with plastic waste completely or by changing it into biomass? Scientists have managed to biodegrade polymers, but the degradation level is slow and incomplete. If the polymer biodegradation technologies were implemented, they would make the plastic recycling process cost effective and environmentally friendly.

Plastic bio cycling holds the promise, but It is hard to state it as planet-saving breakthrough at this point of time. It is not right to say that problem is solved when it hasn't actually. Otherwise, it can undermine the relationships between science and society.

A cocktail of enzymes could handle the problems to fully degrade not only PET but other plastics with different types of chemical bonds. Retrieving the genes for highly active enzymes and implementing them in the production line, would significantly reduce the plastic pollution and could be appreciated as highly rewarding task in the Micro- biotechnology field.

\section{Bibliography}

1. Akutsu Y., et al. "Purification and properties of a polyester polyurethane-degrading enzyme from Comamonas acidovorans TB-35". Applied and Environmental Microbiology 64 (1998): 62-67.

2. Aneta K., et al. "Degradation of plastics and plastics- degrading bacteria in cold marine habitats". Applied Microbiology and Biotechnology 102.18 (2018).

3. Association Française de Normalisation (AFNOR). Plasticsassessment of oxobiodegradability of polyolefinic materials in the form of films-methods and requirements 51.808 (2012):1-25.

4. ASTM International Standard test method for weight attrition of plastic materials in the marine environment by open system aquarium incubations (2012): 1-4.

5. Biffinger JC., et al. "The applicability of Impranil® DLN for gauging the biodegradation of polyurethanes". Polymer Degradation and Stability 120 (2015): 178185.

6. Bollinger A., et al. "The biotechnological potential of marine bacteria in the novel lineage of Pseudomonas pertucinogena". Microbial Biotechnology (2018).

7. Brandon AM., et al. "Biodegradation of polyethylene and plastic mixtures in mealworms (larvae of Tenebrio molitor) and effects on the gut microbiome". Environmental Science and Technology 52 (2018): 6526-6533.

8. Bryant JA., et al. "Diversity and activity of communities inhabiting plastic debris in the North Pacific Gyre". mSystems 1.3 (2016): e00024-e00016.

9. Crabo AG., et al. "Structure and biochemistry of phenylacetaldehyde dehydrogenase from the Pseudomonas putida S12 styrene catabolic pathway". Archives of Biochemistry and Biophysics 616 (2017): 47.

10. Danso D., et al. "New insights into the function and global distribution of polyethylene terephthalate (PET)-degrading bacteria and enzymes in marine and terrestrial metagenomes". Applied and Environmental Microbiology 84 (2018): e02773e02717.

11. Derraik JG. "The pollution of the marine environment by plastic debris: a review". Marine Pollution Bulletin 44.9 (2002): 842-852.

12. De Tender CA., et al. "Bacterial community profiling of plastic litter in the Belgian part of the North Sea". Environmental Science and Technology 49.16 (2015): 9629-9638.

13. Dominik Danso., et al. "Streit". Plastics: Environmental and Biotechnological Perspective on Microbial Degradation (2019).

14. Duke University. Breaking down plastic degradation (2019).

15. Erin Blakemore. Chaw Down on a Plastic-Eating Fungus (2016).

16. Eubeler JP., et al. "Environmental biodegradation of synthetic polymers I. Test methodologies and procedures". Trends in Analytical Chemistry 28 (2009): 1057-1072.

17. Eubeler JP., et al. "Environmental biodegradation of synthetic polymers II. Biodegradation of different polymer groups". Trends in Analytical Chemistry 29 (2009): 84-100.

18. Geng Y., et al. "Biodegradation of isopropanol by a solventtolerant Paracoccus denitrificans strain". Biotechnology and Applied Biochemistry 45 (2015): 4919.

19. Geyer Ronald., et al. "Production, use, and fate of all plastics ever made". Science Advances 3.7 (2017): e1700782. 
New Initiatives are to be Enforced to Execute More Research on Thorough Degradation of Global Polymers Using Diversified Microbes/ Enzymes in the Eco-Friendly Way

20. Hajighasemi M., et al. "Screening and characterization of novel polyesterases from environmental metagenomes with high hydrolytic activity against synthetic polyesters". Environmental Science and Technology 52 (2018): 12388-12401.

21. Harrison P., et al. "Interactions between microorganisms and marine microplastics: a call for research". Marine Technology Society Journal 45.2 (2011): 12-20.

22. Harrison, J. P., et al. "Biodegradability standards for carrier bags and plastic films in aquatic environments: a critical review". Royal Society Open Science 5 (2018): 171792.

23. Howard GT and Blake RC. "Growth of Pseudomonas fluorescens on a polyester-polyurethane and the purification and characterization of a polyurethanase-protease enzyme". International Biodeterioration and Biodegradation 42 (1998): 213-220.

24. Howard GT., et al. "Effect of insertional mutations in the pueA and pueB genes encoding two polyurethanases in Pseudomonas chlororaphis contained within a gene cluster". Journal of Applied Microbiology 103 (2007): 2074-2083.

25. Ian Sample. Plastic - eating worms could help wage war on waste (2017)

26. Itzel Gaytan., et al. "Degradation of Recalcitrant Polyurethane and Xenobiotic Additives by a Selected Landfill Microbial Community and Its Biodegradative Potential Revealed by Proximity Ligation-Based Metagenomic Analysis". Frontiers in Microbiology (2020).

27. Jesse P Harrison., et al. Biodegradability standards for carrier bags and plastic films in aquatic environments: a critical review (2018).

28. Kakudo S., et al. "Nylon oligomer degradation gene, nylC, on plasmid pOAD2 from a Flavobacterium strain encodes endotype 6-aminohexanoate oligomer hydrolase: purification and characterization of the nylC gene product". Applied and Environmental Microbiology 59 (1993): 3978.

29. Kanagawa K., et al. "Characterization of the 6-aminohexanoate-dimer hydrolase from Pseudomonas sp. NK87". Journal of General Microbiology 139 (1993): 787-795.

30. Krueger MC., et al. "Potential of wood-rotting fungi to attack polystyrene sulfonate and its depolymerisation by Gloeophyllum trabeum via hydroquinone-driven fenton chemistry". PLoS One 10 (2015): e0131773.
31. Lucas N., et al. "Polymer biodegradation: mechanisms and estimation techniques. a review". Chemosphere 73 (2008): 429442.

32. MartinezMartinez M., et al. "Determinants and prediction of esterase substrate promiscuity patterns". ACS Chemical Biology 13 (2018): 225-234.

33. Negoro S., et al. "A new nylon oligomer degradation gene (nylC) on plasmid pOAD2 from a Flavobacterium sp". Journal of Bacteriology 174 (1992): 7948-7953.

34. Nowlin T. "Global polyethylene business overview”. (2014) In Nowlin TE (ed), Business and technology of the global polyethylene industry. Wiley-VCH, Weinheim, Germany (2014).

35. Oelschlägel M., et al. "Styrene oxide isomerase of Rhodococcus opacus $1 \mathrm{CP}$, a highly stable and considerably active enzyme". Applied and Environmental Microbiology 78 (2012): 43304337.

36. O'Leary ND., et al. "Functional characterization of a StyS sensor kinase reveals distinct domains associated with intracellular and extracellular sensing of styrene in P. putida CA-3". Bioengineered 5 (2014): 114-122.

37. O'Leary ND., et al. "Genetic characterization of accumulation of polyhydroxyalkanoate from styrene in Pseudomonas putida CA-3". Applied and Environmental Microbiology 71 (2005): 4380-4387.

38. Paco A., et al. "Biodegradation of polyethylene microplastics by the marine fungus Zalerion maritimum". Science of the Total Environment 586 (2017): 10-15.

39. Palm GJ., et al. "Structure of the plastic-degrading Ideonella sakaiensis MHETase bound to a substrate". Nature Communications 10 (2019): 1717.

40. Palmer R. "Polyamides, plastics". In Encyclopedia of polymer science and technology. Wiley, Hoboken, NJ.Negoro S, Taniguchi T, Kanaoka M, Kimura H, Okada H. 1983. Plasmid-determined enzymatic degradation of nylon oligomers". Journal of Bacteriology 155 (2001): 22-31.

41. Plastics Europe, plastics-the facts 2018: "an analysis of European plastics production, demand and waste data". PlasticsEurope, Brussels, Belgium (2018).

42. Sowmya HV., et al. "Degradation of polyethylene by Penicillium simplicissimum isolated from local dumpsite of Shivamogga district". Environment, Development and Sustainability 17 (2015): 731-745. 
43. Syranidou E., et al. "Biodegradation of weathered polystyrene films in seawater microcosms". Scientific Reports 7 (2017): 17991.

44. Takehara I., et al. "Metabolic pathway of 6-aminohexanoate in the nylon oligomer-degrading bacterium Arthrobacter sp. KI72: identification of the enzymes responsible for the conversion of 6-aminohexanoate to adipate". Applied Microbiology and Biotechnology 102 (2018): 801-814.

45. Tekman MB., et al. 1,510 species are affected by litter, Alfred Wegener Institute Helmholtz Centre for Polar and Marine Research (2017).

46. Tischler D. "Microbial styrene degradation". Springer International Publishing, Cham, Switzerland (2015): 7-22.

47. Weber C., et al. "Polyethylene bio-degradation by caterpillars?" Current Biology 27 (2017): R744-R745.

48. Yang Y., et al. "Biodegradation and mineralization of polystyrene by plastic-eating mealworms: Part 1 . Chemical and physical characterization and isotopic tests". Environmental Science and Technology 49 (2015): 12080-12086.

49. Yang Y., et al. "Complete genome sequence of Bacillus sp. YP1, a polyethylene-degrading bacterium from waxworm's gut". Journal of Biotechnology 200 (2015): 77-78.

\section{Assets from publication with us}

- Prompt Acknowledgement after receiving the article

- Thorough Double blinded peer review

- Rapid Publication

- Issue of Publication Certificate

- High visibility of your Published work

Website: https://www.actascientific.com/

Submit Article: https://www.actascientific.com/submission.php

Email us: editor@actascientific.com

Contact us: +919182824667

Citation: Anju Kaushal. "New Initiatives are to be Enforced to Execute More Research on Thorough Degradation of Global Polymers Using Diversified Microbes/ Enzymes in the Eco-Friendly Way". Acta Scientific Microbiology 3.4 (2020): 97-108. 